\title{
ON PARTIAL BARYCENTRIC SUBDIVISION
}

\author{
SARFRAZ AHMAD ${ }^{1}$ AND VOLKMAR WELKER
}

\begin{abstract}
The $l^{\text {th }}$ partial barycentric subdivision is defined for a $(d-1)$-dimensional simplicial complex $\Delta$ and studied along with its combinatorial, geometric and algebraic aspects. We analyze the behavior of the $f$-and $h$-vector under the $l^{\text {th }}$ partial barycentric subdivision extending previous work of Brenti and Welker on the standard barycentric subdivision - the case $l=1$. We discuss and provide properties of the transformation matrices sending the $f$ - and $h$-vector of $\Delta$ to the $f$ - and $h$-vector of its $l^{\text {th }}$ partial barycentric subdivision. We conclude with open problems.
\end{abstract}

\section{INTRODUCTION}

For a $(d-1)$-dimensional simplicial complex $\Delta$ on the ground set $V$ the barycentric subdivision $\operatorname{sd}(\Delta)$ of $\Delta$ is the simplicial complex on the ground set $V \backslash\{\emptyset\}$ with simplices the flags $A_{0} \subset A_{1} \subset \cdots \subset A_{i}$ of elements $A_{j} \in \Delta \backslash\{\emptyset\}, 0 \leq j \leq i$. For $1 \leq l \leq d$, we define the $l^{\text {th }}$ partial barycentric subdivision of $\Delta$. This is a geometric subdivision, in the sense of 8 , such that $\mathrm{sd}^{l-1}(\Delta)$ is a refinement of $\operatorname{sd}^{l}(\Delta), \operatorname{sd}^{d}(\Delta)=\Delta$ and $\operatorname{sd}^{1}(\Delta)=\operatorname{sd}(\Delta)$. Roughly speaking, the $l^{\text {th }}$ partial barycentric subdivision arises when only the simplices of dimension $\geq l$ are barycentrically subdivided. In the paper, we provide a detailed analysis of the effect of the $l^{\text {th }}$ barycentric subdivision operation on the $f$ - and $h$-vector of a simplicial complex. Most enumerative results will be related to refinements of permutation statistics for the symmetric group. Our results extend the results from [3] for the case $l=1$. We refer the reader also to [4] and [7] for more detailed information in that case.

The paper is organized as follows. We start in Section 2 with geometric and combinatorial descriptions of the $l^{\text {th }}$ partial barycentric subdivision and its implications on the generators of the Stanley-Reisner ideal of the complex. In Section 3 we study the enumerative combinatorics of the $l^{\text {th }}$ partial barycentric subdivision. In particular, we relate in Lemma 3.1 and Theorem 3.4 the effect of the $l^{\text {th }}$ barycentric subdivision on the $f$-and $h$-vector of the simplicial complex $\Delta$ to a permutation statistics refining the descent statistics. In Section 4 we analyze the transformation matrices sending the $f$-and $h$-vector of the simplicial complex $\Delta$ to the corresponding vector for the $l^{\text {th }}$ barycentric subdivision. We show that both maps are diagonizable and provide the eigenvalue structure. Note that by general facts the two matrices are similar. The main result of this section, Theorem 4.10, shows that the eigenvector corresponding to the highest eigenvalue of the $h$-vector transformation can be

\footnotetext{
Key words and phrases. Barycentric subdivision, $f$-vector, $h$-vector.

${ }^{1}$ Partially supported by Higher Education Commission of Pakistan under the program HEC post doctoral fellowship phase II(batch IV), ref 2-4(45)/PDFP/HEC/2010/2.
} 
chosen such that it is of the form $\left(0, b_{1}, \ldots, b_{d-1}, 0\right)$ for strictly positive numbers $b_{i}$, $1 \leq i \leq d-1$. In Section 5 we present some open problems. We ask for explicit descriptions of the eigenvectors and then shift the focus to the local $h$-vector which has been introduced by Stanley [8]. The local $h$-vector is a measure for the local effect of a subdivision operation. In particular, general results by Stanley, predict that the local $h$-vector for the $l^{\text {th }}$ partial barycentric subdivision is non-negative. For $l=1$ the local $h$-vector was computed by Stanley in terms of the excedance statistics on derangements. We exhibit some computations and possible approaches to the local $h$-vector for the $l^{\text {th }}$ barycentric subdivision in general.

\section{The $l^{\mathrm{TH}}$ PARTIAL BARYCENTRIC SUBDIVISION}

2.1. Geometric definition. We first give a geometric definition of the $l^{\text {th }}$ partial barycentric subdivision. For that we recall some basic facts about the reflection arrangement of the symmetric group $S_{d}$ permuting the $d$ letters from $[d]:=$ $\{1,2, \ldots, d\}$. The reflection arrangement $\mathcal{B}_{d}$ in $\mathbb{R}^{d}$ of the symmetric group $S_{d}$ consists of the hyperplanes $H_{i j}=\left\{\left(x_{1}, \ldots, x_{d}\right) \in \mathbb{R}^{d}: x_{i}-x_{j}=0\right\}, 1 \leq i<j \leq d$. To each permutation $w \in S_{d}$ there corresponds a region $R_{w}$ of $\mathcal{B}_{d}$ given by

$$
R_{w}=\left\{\left(\lambda_{1}, \ldots, \lambda_{d}\right) \in \mathbb{R}^{d}: \lambda_{w(1)}>\lambda_{w(2)}>\cdots>\lambda_{w(d)}\right\} .
$$

Hence the number of regions of $\mathcal{B}_{d}$ is $d$ !. We write $R_{w,+}$ for the intersection of $R_{w}$ with $\mathbb{R}_{>0}^{d}$. It is easily seen that geometrically the closure of $R_{w,+}$ is a simplicial cone.

The intersection of the closures of the cones $R_{w,+}, w \in S_{d}$, and the standard $(d-1)$ simplex $\Delta_{d-1}=\left\{\left(\lambda_{1}, \ldots, \lambda_{d}\right) \in \mathbb{R}^{d} \mid \lambda_{1}+\cdots \lambda_{d}=1, x_{i} \geq 0,1 \leq i \leq d\right\}$ induces a simplicial decomposition of $\Delta_{d-1}$. This decomposition is called the barycentric subdivision of $\Delta_{d-1}$ and is denoted by $\operatorname{sd}\left(\Delta_{d-1}\right)$. We are interested in a sequence $\operatorname{sd}^{l}\left(\Delta_{d-1}\right), 1 \leq i \leq d$, of simplicial subdivisions of the simplex, which have the property that $\operatorname{sd}^{1}\left(\Delta_{d-1}\right)=\operatorname{sd}\left(\Delta_{d-1}\right)$ and $\operatorname{sd}^{l-1}\left(\Delta_{d-1}\right)$ is a refinement of $\operatorname{sd}^{l}\left(\Delta_{d-1}\right)$. For $1 \leq l \leq d$, we set $S_{d}^{l}$ to be the set of permutations $w \in S_{d}$ for which $w(1)>$ $\cdots>w(l)$. We define the $l$-cone $R_{w}^{l}$ of a $w \in S_{d}^{l}$ to be

$$
R_{w}^{l}=\left\{\left(\lambda_{1}, \ldots, \lambda_{d}\right) \in \mathbb{R}^{d}: \lambda_{w(1)}, \ldots, \lambda_{w(l)}>\lambda_{w(l+1)}>\cdots>\lambda_{w(d)}\right\} .
$$

Clearly $R_{w}^{l}$ is a cone. We write $R_{w,+}^{l}$ for the intersection of $R_{w}^{l}$ with $\mathbb{R}_{\geq 0}^{d}$. Again the closure of $R_{w,+}^{l}$ is a simplicial cone which is the union of all closures of the $R_{v,+}$ for $v \in S_{d}$ such that $v(i)=w(i)$ for $l+1 \leq i \leq n$. We call the simplicial decomposition induced by the collection of all $R_{w,+}^{l}$ for $w \in S_{d}^{l}$ on $\Delta_{d+1}$ the $l^{\text {th }}$ partial barycentric subdivision of $\Delta_{d-1}$ and denote it by $\operatorname{sd}^{l}\left(\Delta_{d-1}\right)$. Obviously, we have that $\operatorname{sd}^{d}\left(\Delta_{d-1}\right)=\Delta_{d-1}, \operatorname{sd}^{1}\left(\Delta_{d-1}\right)=\operatorname{sd}\left(\Delta_{d-1}\right)$ and $\operatorname{sd}^{l-1}\left(\Delta_{d-1}\right)$ is a refinement of $\operatorname{sd}^{l}\left(\Delta_{d-1}\right)$. If $l>d$ then we set $\operatorname{sd}^{l}\left(\Delta_{d-1}\right)=\Delta_{d-1}$. For a $(d-1)-$ dimensional simplicial complex $\Delta$ on the vertex set $V=[n]$ its $l^{\text {th }}$ partial barycentric subdivision is the complex $\operatorname{sd}^{l}(\Delta)$ which is the subdivision of $\Delta$ obtained by replacing each simplex by its $l^{\text {th }}$ partial subdivision. Roughly speaking this means that we cone all $(k-1)$-faces of $\Delta$ over their barycenters for all $l \leq k$.

By construction the number of cones $R_{w}^{l}, w \in S_{d}^{l}$, is $\frac{d !}{(d-l) !}=d \cdot(d-1) \cdots(d-l+1)$. Next, we want to get a better understanding of the facial structure of $\operatorname{sd}^{l}\left(\Delta_{d-1}\right)$. 
We have already seen that the $(d-1)$-dimensional faces are in bijection with the permutations in $S_{d}^{l}$. We turn this description into a description by combinatorial objects that are more suitable for studying all faces of $\operatorname{sd}^{l}\left(\Delta_{d-1}\right)$. We identify a permutation $w \in S_{d}^{l}$ with a formal chain

$$
w(1), w(2), \ldots, w(l)>w(l+1)>\cdots>w_{d}
$$

and this chain in turn with $A_{0} \subset A_{1} \subset \cdots \subset A_{d-l}$ for $A_{i}=\{w(1), \ldots, w(l), \ldots, w(l+$ $i)\}$.

Using this chain description, a 2-dim face of $\operatorname{sd}^{l}\left(\Delta_{2}\right)$ corresponding to $w=123$ is either $\{1,2,3\}$ for $l=3$ or $\{1,2\} \subset\{1,2,3\}$ for $l=2$ or $\{1\} \subset\{1,2\} \subset\{1,2,3\}$ for $l=1$.

More generally, the $(i-1)$-faces of $\operatorname{sd}^{l}(\Delta)$ are indexed by chains $A_{0} \subset A_{1} \subset \cdots \subset$ $A_{r}$ for which:

(C1) $0 \leq \# A_{0} \leq l$

(C3) $l+1 \leq \# A_{1}$.

(C2) $\# A_{0}+r=i$,

(C4) $A_{r} \in \Delta$.

At the beginning of Section 3 we will further reformulate this description in terms of yet another combinatorial objects.

Geometrically, the face of $\operatorname{sd}^{l}\left(\Delta_{d-1}\right)$ corresponding to $A_{0} \subset A_{1} \subset \cdots \subset A_{0}$ is the set of points $\left(\lambda_{1}, \ldots, \lambda_{d}\right) \in \Delta_{d-1}$ for which

(i) We have $\lambda_{i}=\lambda_{j}$ if $i, j \in A_{s} \backslash A_{s-1}$ for some $1 \leq s \leq r$.

(ii) We have $\lambda_{i}>\lambda_{j}$ if $i \in A_{s}$ and $j \in A_{t}$ for some $0 \leq s<t \leq r$.

(iii) We have $\lambda_{i}=0$ if $i \notin A_{r}$.

In particular, a vertex $v$ of the $l^{\text {th }}$ partial barycentric subdivision $\operatorname{sd}^{l}(\Delta)$ of a $(d-1)$-dimensional simplicial complex $\Delta$ either belongs to the vertex set of $\Delta$ or can be identified with an $(m-1)$-face $v=\left\{v_{i_{1}}, v_{i_{2}}, \ldots, v_{i_{m}}\right\} \in \Delta$ of $\Delta$ for some $l \leq m \leq d$.

2.2. Algebraic aspects. Let $\Delta$ be a $(d-1)$-dimensional simplicial complex on vertex set $[n]$. Let $k$ be a field and $R=k\left[x_{1}, \ldots, x_{n}\right]$ be the polynomial ring in $n$ variables. The Stanley-Reisner ideal $I_{\Delta}$ is the ideal of $R$ generated by the squarefree monomials $\prod_{i \in \mathcal{N}} x_{i}$ whose index set $\mathcal{N}$ is a non-face of $\Delta$. A non-face $\mathcal{N} \notin \Delta$ is called a minimal non-face of $\Delta$ if no proper subset of $\mathcal{N}$ is a non-face of $\Delta$. It is easily seen that the generators in the unique minimal monomial generating set of the Stanley-Reisner ideal correspond to the minimal non-faces of $\Delta$. The quotient $R / I_{\Delta}$ is called the Stanley-Reisner ring or face ring and is denoted by $k[\Delta]$.

Let $\operatorname{sd}^{l}(\Delta)$ be the $l^{\text {th }}$ partial barycentric subdivision of $\Delta$, where $0 \leq l \leq d-1$. Similarly, let $I_{\mathrm{sd}^{l}(\Delta)}$ and $k\left[\operatorname{sd}^{l}(\Delta)\right]$ be the Stanley-Reisner ideal and face ring of $\operatorname{sd}^{l}(\Delta)$, respectively. Let $V_{l}=[n] \cup B_{l}$ be the set of vertices of $\operatorname{sd}^{l}(\Delta)$, where $B_{l}=\left\{b_{1}, \ldots, b_{l}\right\}$ is the set of barycenters of the faces of $\Delta$ whose dimension ranges between $l$ and $d-1$.

Lemma 2.1. Let $\Delta$ be a $(d-1)$-dimensional simplicial complex on ground set $[n]$. For $1 \leq l \leq d$ let $\mathcal{N}$ be a subset of the vertex set of $\operatorname{sd}^{l}(\Delta)$. In case $\mathcal{N}$ is a minimal non-face of $\operatorname{sd}^{l}(\Delta)$ then either $\mathcal{N} \subseteq[n]$ and $\mathcal{N}$ is a minimal non-face of $\Delta$ or $|\mathcal{N}|=2$. 
Proof. If $\mathcal{N} \subset[n]$ and a minimal non-face of $\Delta$, then we are done. Since two vertices of $\Delta$ are connected by an edge in $\operatorname{sd}^{l}(\Delta)$ if and only if they are connected by an edge in $\Delta$ it follows that any subset of $[n]$ that is a minimal non-face of $\operatorname{sd}^{l}(\Delta)$ must be a minimal non-face of $\Delta$. Now, suppose there exists at least one vertex $b \in \mathcal{N} \backslash[n]$ and $|\mathcal{N}| \neq 2$. If $|\mathcal{N}|=1$ then $\mathcal{N}=\{b\}$ but $b$ is a vertex and hence a face. This leads to a contradiction and we are left with the case $|\mathcal{N}| \geq 2$. Let $\mathcal{F} \in \operatorname{sd}^{l}(\Delta)$ be a facet such that $b \in \mathcal{F}$. Then there exists at least one vertex $v \in \mathcal{N} \backslash \mathcal{F}$, otherwise $\mathcal{N}$ is no more a non-face. But by construction the edge $\{b, v\}$ is not an edge in $\operatorname{sd}^{l}(\Delta)$. Thus $\left\{b_{j}, v\right\} \subset \mathcal{N}$ from which $\left\{b_{j}, v\right\}=\mathcal{N}$ follows.

Now we have the following Proposition:

Proposition 2.2. Let $\Delta$ be a $(d-1)$-dimensional simplicial complex on ground set $[n]$. Then for $1 \leq l \leq d-1$ the Stanley-Reisner ideal $I_{\mathrm{sd}^{l}(\Delta)}$ is generated by square free monomial ideals of degree at most $l+1$.

Proof. Suppose there exists some generator $u \in I_{\mathrm{sd}^{l}(\Delta)}$ with degree strictly larger than $l+1$. Then there exists a minimal non-face $\mathcal{N} \subset \operatorname{sd}^{l}(\Delta)$ such that $|\mathcal{N}|>l+1$. Thus by Lemma 2.1 it follows that $\mathcal{N} \subset[n]$. But in $\operatorname{sd}^{l}(\Delta)$, we have coned all faces $\mathcal{F} \subseteq[n]$ of dimension $\geq l$ over their barycenters. Thus there does not exist any non-face of dimension greater than $l$. Therefore, $|\mathcal{N}| \leq l+1$, a contradiction.

\section{3. $f$-VECTOR AND $h$-VECTOR TRANSFORMATION}

In this section we study the transformation maps sending the $f$-and $h$-vector of a simplicial complex $\Delta$ to the $f$ - and $h$-vector of the $l^{\text {th }}$ partial barycentric subdivision of $\Delta$.

We consider set systems $B=\left|B_{0}\right| B_{1}|\ldots| B_{r} \mid$ such that $B_{1}, \ldots, B_{r} \neq \emptyset$ and $B_{s} \cap B_{t}=$ $\emptyset$ for $0 \leq s<t \leq r$. Despite the fact that $B_{0}$ can be empty we call such a system an ordered set partition. We write $R(j, i, l)$ for the number of such ordered set partitions $B=\left|B_{0}\right| B_{1}|\ldots| B_{r} \mid$ for which

(P1) $B_{0} \cup \cdots \cup B_{r}=[j]$

(P2) If $r \geq 1$ then $\#\left(B_{0} \cup B_{1}\right) \geq l+1$

(P3) $\# B_{0}+r=i$.

and call $R(j, i, l)$ the restricted Stirling number for the parameters $j, i, l$. For $l=0$ by $\#\left(B_{0} \cup B_{1}\right) \geq l+1=1$ it follows from $B_{1} \neq \emptyset$ that $B_{0}=\emptyset$. Hence $r=i$ and $\left|B_{1}\right| \cdots\left|B_{i}\right|$ is a usual ordered set partition of the $j$-element set $B_{1} \cup \cdots \cup B_{i}$ into $i$ (non-empty) blocks. Thus $R(j, i, 0)=i ! S(j, i)$, where $S(i, j+1)$ is the Stirling number of second kind.

Recall that the $f$-vector $f^{\Delta}=\left(f_{-1}^{\Delta}, \ldots, f_{d-1}^{\Delta}\right)$ of a $(d-1)$-dimensional simplicial complex is the vector with its $i^{\text {th }}$ entry $f_{i}^{\Delta}$ counting the $i$-dimensional faces of $\Delta$. Using this notation, we have the following lemma. 
Lemma 3.1. Let $\Delta$ be a $(d-1)$-dimensional simplicial complex with $f$-vector $f^{\Delta}=\left(f_{-1}^{\Delta}, \ldots, f_{d-1}^{\Delta}\right)$. Then

$$
f_{i-1}^{\mathrm{sd}^{l}(\Delta)}=\sum_{j=0}^{d} f_{j-1}^{\Delta} \cdot R(j, i, l) .
$$

Proof. Let $A_{0} \subset A_{1} \subset \cdots \subset A_{r}$ be an $(i-1)$-dimensional face of $\operatorname{sd}^{l}(\Delta)$. Then by (C2) we have $i=\# A_{0}+r$. We set $j=\# A_{r}$ and assume without loss of generality that $A_{r}=[j]$. We define $B_{0}=A_{0}$ and $B_{s}=A_{s} \backslash A_{s-1}$ for $1 \leq s \leq r$. Then $i=\# B_{0}+r$ and $j=\#\left(B_{0} \cup \cdots \cup B_{r}\right)$. If $r \geq 1$ then by (C3) \# $A_{1}=\#\left(B_{0} \cup B_{1}\right) \geq l+1$. Hence $\left|B_{0}\right| \cdots\left|B_{r}\right|$ satisfies (P1)-(P3). Conversely, if $\left|B_{0}\right| \cdots\left|B_{r}\right|$ satisfies (P2)-(P3) and $\left(B_{0} \cup \cdots \cup B_{r}\right) \in \Delta$ then one easily checks that for $A_{s}=B_{0} \cup \cdots \cup B_{s}, 0 \leq s \leq r$, the chain $A_{0} \subset \cdots \subset A_{r}$ satisfies (C1)-(C4).

Thus for any $(j-1)$-dimensional face $F$ of $\Delta$ on gets $R(j, i, l)$ faces $A_{0} \subset \cdots \subset A_{r}$ of dimension $i-1$ in $\operatorname{sd}^{l}(\Delta)$ with $F=A_{r}$.

Next we study the transformation of the $h$-vector. Recall that the $h$-vector of a $(d-1)$-dimensional simplicial complex $\Delta$ is the integer vector $h^{\Delta}=\left(h_{0}^{\Delta}, \ldots, h_{d}^{\Delta}\right)$ for which $\sum_{i=0}^{d} f_{i-1}^{\Delta} x^{d-i}=\sum_{i=0}^{d} h_{i}^{\Delta} x^{d-i}$, where $x$ is some indeterminate. For a permutation $\sigma \in S_{d}$ we denoted by

$$
\mathrm{D}(\sigma)=\{i \in[d-1] \mid \sigma(i)>\sigma(i+1)\}
$$

its decent set and write $\operatorname{des}(\sigma):=\# \mathrm{D}(\sigma)$ for its number of descents. Following [3] for $d \geq 1$ and integers $i$ and $j$ we denote by $A(d, i, j)$ the number of permutations $\sigma \in S_{d}$ such that $\sigma(1)=j$ and $\operatorname{des}(\sigma)=i$. In particular, $A(d, i, j)=0$ if $i \leq-1$ or $i \geq d$.

In the sequel, we define a refinement of the preceding statistics suitable for the study of our $h$-vector transformation. By definition of $S_{d}^{l}$ we have the following strictly increasing chain of subgroups:

$$
S_{d+1}^{d-1} \subset S_{d+1}^{d-2} \subset \cdots \subset S_{d+1}^{2} \subset S_{d+1}^{1}=S_{d+1} .
$$

We define the $l$-descent set $D^{l}(\sigma)$ of a permutation $\sigma \in S_{d}^{l}$ as follows:

Definition 3.2. A number $i \in[d-1]$ belongs to the $l$-descent set $D^{l}(\sigma)$ of $\sigma \in S_{d}^{l}$, if $i$ satisfies one of the following two conditions.

(1) $i \in[l]$ and $\sigma(i)>\sigma(l+1)$,

(2) $i \in[d-1] \backslash[l]$ and $\sigma(i)>\sigma(i+1)$.

We write $\operatorname{des}^{l}(\sigma)=\left|D^{l}(\sigma)\right|$ for the number of $l$-descents of a permutation $\sigma \in S_{d}^{l}$. Note that for $l=1$ condition (1) is equivalent to having a decent in position one and therefore $D^{1}(\sigma)$ is just the usual descent set of $\sigma$.

Example 3.3. Let $\sigma_{1}, \sigma_{2}, \sigma_{3} \in S_{6}^{4}$, such that, $\sigma_{1}=(\underline{4,3,2,1}, 6,5), \sigma_{2}=(\underline{6,5,2,1}, 3,4)$ and $\sigma_{3}=(6,4,3,2,5,1)$. Then

$$
\begin{array}{ccc}
D^{4}\left(\sigma_{1}\right)=\{5\} & D^{4}\left(\sigma_{2}\right)=\{1,2\} & D^{4}\left(\sigma_{3}\right)=\{1,5\} \\
\operatorname{des}^{4}\left(\sigma_{1}\right)=1 & \operatorname{des}^{4}\left(\sigma_{2}\right)=2 & \operatorname{des}^{4}\left(\sigma_{3}\right)=2 .
\end{array}
$$


For all $d \geq 1,1 \leq l \leq d-1$ and all integers $i$ and $j$ we denote by $A(d, i, j, l)$ the number of permutations $\sigma \in S_{d}^{l}$ such that $\operatorname{des}^{l}(\sigma)=i$ and $\sigma(d+1)=j$. Note that $A(d, i, j, l)=0$ if $i \leq-1$ or $i \geq d$.

The following is our first main result. The case $l=1$ was treated in [3, Thm. 1].

Theorem 3.4. Let $\Delta$ be a $(d-1)$-dimensional simplicial complex. Then

$$
h_{j}^{\mathrm{sd}^{l}(\Delta)}=\sum_{\mu=0}^{d} A(d+1, j, d+\mu-1, l) h_{\mu}^{\Delta}
$$

for $1 \leq l \leq d-1$ and $0 \leq j \leq d$.

Proof. For all $0 \leq j \leq d$, we have:

$$
\begin{aligned}
h_{j}^{\mathrm{sd}^{l}(\Delta)} & =\sum_{i=0}^{j}\left(\begin{array}{l}
d-i \\
j-i
\end{array}\right)(-1)^{j-i} f_{i-1}^{\mathrm{sd}^{l}(\Delta)} \\
& =\sum_{i=0}^{j}\left(\begin{array}{l}
d-i \\
j-i
\end{array}\right)(-1)^{j-i} \sum_{k=0}^{d} f_{k-1}^{\Delta} R(k, i, l) \\
& =\sum_{i=0}^{j} \sum_{k=0}^{d}\left(\begin{array}{l}
d-i \\
j-i
\end{array}\right)(-1)^{j-i} R(k, i, l) \sum_{\mu=0}^{k}\left(\begin{array}{l}
d-\mu \\
d-k
\end{array}\right) h_{\mu}^{\Delta} \\
& =\sum_{\mu=0}^{d}\left(\sum_{k=0}^{d} \sum_{i=0}^{j}(-1)^{j-i}\left(\begin{array}{l}
d-i \\
j-i
\end{array}\right)\left(\begin{array}{l}
d-\mu \\
d-k
\end{array}\right) R(k, i, l)\right) h_{\mu}^{\Delta} .
\end{aligned}
$$

Fix a permutation $\sigma \in S_{d}^{l}$ and let $\mathrm{D}^{l}(\sigma)=\left\{s_{1}, \ldots, s_{k}\right\} \subseteq[d]$ with $s_{1}<\cdots<s_{k}$ its descent set. We define $p_{\sigma}$ be 0 if there is no $s_{p_{\sigma}} \in[l]$ for which $\sigma\left(s_{p_{\sigma}}\right)>\sigma(l+1)$ and to be the maximal number $p_{\sigma} \in[k]$ for which $s_{p_{\sigma}} \in[l]$ and $\sigma\left(s_{p_{\sigma}}\right)>\sigma(l+1)$. Thus by definition of an $l$-descent any set $S$ that can arise as the descent set of a permutation $\sigma \in S_{d}^{l}$ is of the form $S=\left\{1, \ldots, p, s_{p+1}, \ldots, s_{k}\right\}$ for some $0 \leq p_{\sigma}<l \leq s_{p}+1$. We want to count

$$
\left\{\begin{array}{l|l}
\sigma \in S_{d+1}^{l} & \begin{array}{l}
\mathrm{D}^{l}(\sigma) \subseteq S, p_{\sigma}=p \\
\sigma(d+1)=d+1-\mu
\end{array}
\end{array}\right\}
$$

First, we count the possibilities to choose $\sigma\left(s_{p+1}+1\right), \ldots, \sigma(d+1)$. In this range a descent is just a usual descent and we have $\left(\begin{array}{c}s_{k} \\ s_{p+1}, s_{p+2}-s_{p+1}, \ldots, s_{k}-s_{k-1}\end{array}\right)\left(\begin{array}{c}d-\mu \\ d-t_{k}\end{array}\right)$ possibilities. Having done this we are left with $s_{p+1}$ elements that we have to arrange accordingly. Let $U$ be this set of elements. Let us consider the options for $\sigma(l+1)$. In order to create no descent in the range of $l+1$ and $s_{p+1}-1$ there have to be at least $s_{p+1}-(l+1)$ elements larger than $\sigma(l+1)$. Hence there can be at most $l$ elements smaller than $\sigma(l+1)$. In order to create no descent between $\sigma(i)$ and $\sigma(l+1)$ for some $p<i<l+1$ there must be at least $l-p$ elements smaller than $\sigma(l+1)$. Hence $\sigma(l+1)$ can only range from the $(l+1-p)^{\text {th }}$ element of $U$ to the $(l+1)^{\text {st }}$ element of $U$. Let $l+1-p \leq i \leq l+1$ and assume $\sigma(l+1)$ is the $i^{\text {th }}$ element of $U$. Then in order to fix the permutation $\sigma$ we have to fix $s_{p+1}-(l+1)$ element larger than 
$\sigma(l+1)$ that will become the images of $\sigma(l+2), \ldots, \sigma\left(s_{p+1}\right)$ in increasing order. For that we have $\left(\begin{array}{c}s_{p+1}-i \\ s_{p+1}-(l+1)\end{array}\right)$ possibilities. Using $l+1 \leq s_{p+1}$ we obtain:

$$
\begin{aligned}
& \#\left\{\begin{array}{l|l}
\sigma \in S_{d+1}^{l} & \begin{array}{l}
\mathrm{D}^{l}(\sigma) \subseteq S, p_{\sigma}=p \\
\sigma(d+1)=d+1-\mu
\end{array}
\end{array}\right\} \\
& =\sum_{i=l+1-p}^{l+1}\left(\begin{array}{c}
s_{p+1}-i \\
s_{p+1}-(l+1)
\end{array}\right)\left(\begin{array}{c}
s_{k} \\
s_{p+1}, s_{p+2}-s_{p+1}, \ldots, s_{k}-s_{k-1}
\end{array}\right)\left(\begin{array}{l}
d-\mu \\
d-t_{k}
\end{array}\right)
\end{aligned}
$$

but,

$$
\begin{gathered}
\sum_{i=l+1-p}^{l+1}\left(\begin{array}{c}
s_{p+1}-i \\
s_{p+1}-(l+1)
\end{array}\right) \\
=\left(\begin{array}{c}
s_{p+1}-l-1+p \\
s_{p+1}-l-1
\end{array}\right)+\left(\begin{array}{c}
s_{p+1}-l-2+p \\
s_{p+1}-l-1
\end{array}\right)+\cdots+\left(\begin{array}{c}
s_{p+1}-l-1 \\
s_{p+1}-l-1
\end{array}\right) \\
=\left(\begin{array}{c}
s_{p+1}-l-1+p+1 \\
s_{p+1}-l-1+1
\end{array}\right)=\left(\begin{array}{c}
s_{p+1}-l+p \\
s_{p+1}-l
\end{array}\right)
\end{gathered}
$$

so,

therefore,

$$
\begin{gathered}
\#\left\{\sigma \in S_{d+1}^{l} \mid \begin{array}{c}
\mathrm{D}^{l}(\sigma) \subseteq S, p_{\sigma}=p \\
\sigma(d+1)=d+1-\mu
\end{array}\right\} \\
=\left(\begin{array}{c}
s_{p+1}-l+p \\
s_{p+1}-l
\end{array}\right)\left(\begin{array}{c}
d-\mu \\
s_{p+1}, s_{p+2}-s_{p+1}, \ldots, s_{k}-s_{k-1}
\end{array}\right)\left(\begin{array}{c}
d-\mu \\
s-s_{k}
\end{array}\right)
\end{gathered}
$$

$$
\begin{gathered}
\sum_{\left\{1 \leq s_{1}<\cdots<s_{k} \leq d\right\}} \sum_{p=0}^{l} \#\left\{\sigma \in S_{d+1}^{l} \mid \begin{array}{c}
\mathrm{D}^{l}(\sigma) \subseteq S, p_{\sigma}=p \\
\sigma(d+1)=d+1-\mu
\end{array}\right\} \\
=\sum_{1 \leq s_{1}<\cdots<s_{k} \leq d} \sum_{p=0}^{l}\left(\begin{array}{c}
s_{p+1}-l+p \\
s_{p+1}-l
\end{array}\right)\left(\begin{array}{c}
s_{k} \\
s_{p+1}, s_{p+2}-s_{p+1}, \ldots, s_{k}-s_{k-1}
\end{array}\right)\left(\begin{array}{c}
d-\mu \\
d-s_{k}
\end{array}\right) \\
=\sum_{j=k}^{d}\left(\begin{array}{c}
d-\mu \\
d-j
\end{array}\right) \sum_{1 \leq s_{1}<\cdots<s_{k-1} \leq j-1} \sum_{p=0}^{l}\left(\begin{array}{c}
s_{p+1}-l+p \\
s_{p+1}-l
\end{array}\right)\left(\begin{array}{c}
j \\
s_{p+1}, s_{p+2}-s_{p+1}, \ldots, j-s_{k-1}
\end{array}\right)
\end{gathered}
$$

For a fixed sequence $s_{1}<\cdots<s_{k}=j$ for which $s_{1}=1, \ldots, s_{p}=p$ and $s_{p+1} \geq l+1$ we set $B_{0}=\left[s_{p}\right]$ for $p \geq 1$ and $B_{0}=\emptyset$ for $p=0$. Also, we set

$$
B_{\omega}=\left[s_{p+\omega-1}+1, s_{p+\omega}\right] \text { for } 1 \leq \omega \leq k-p .
$$

Then $B_{0} \cup \cdots \cup B_{r}=[j]$ which implies (P1). If $r \geq 1$ then $\#\left(B_{0} \cup B_{1}\right)=\# B_{0}+\# B_{1} \geq$ $p+(l+1-p)=l+1$ implying $(\mathrm{P} 2)$. Finally $\# B_{0}+r=p+(k-p)=k$ shows $(\mathrm{P} 3)$.

For notational convenience we set $s_{0}=0$. So for $r=k-p$,

$$
\sum_{1 \leq s_{1}<\cdots<s_{k-1} \leq j-1} \sum_{p=0}^{l}\left(\begin{array}{c}
s_{p+1}-l+p \\
s_{p+1}-l
\end{array}\right)\left(\begin{array}{c}
j \\
s_{p+1}, s_{p+2}-s_{p+1}, \ldots, j-s_{k-1}
\end{array}\right)
$$

counts the number of $B=\left|B_{0}\right| B_{1}|\ldots| B_{r} \mid$ of $[j]$ satisfying (P1) - (P3). Therefore (1) equals $R(j, k, l)$. 
Using this result we have,

$$
\begin{aligned}
& \sum_{S \subseteq[d], \# S=k} \#\left\{\begin{array}{l|l}
\sigma \in S_{d+1}^{l} & \begin{array}{l}
\mathrm{D}^{l}(\sigma) \subseteq S, \\
\sigma(d+1)=d+1-\mu
\end{array}
\end{array}\right\} \\
& =\sum_{j=k}^{d}\left(\begin{array}{l}
d-\mu \\
d-j
\end{array}\right) R(j, k, l)
\end{aligned}
$$

therefore,

$$
\begin{aligned}
& \sum_{k=0}^{d} \sum_{i=0}^{j}(-1)^{j-i}\left(\begin{array}{l}
d-i \\
j-i
\end{array}\right)\left(\begin{array}{l}
d-\mu \\
d-k
\end{array}\right) R(k, i, l) \\
& =\sum_{i=0}^{j}(-1)^{j-i}\left(\begin{array}{l|l}
d-i \\
j-i
\end{array}\right) \sum_{\{S \subseteq[d], \# S=i\}} \#\left\{\begin{array}{ll}
\sigma \in S_{d+1}^{l} & \begin{array}{l}
\mathrm{D}^{l}(\sigma) \subseteq S, \\
\sigma(d+1)=d+1-\mu
\end{array}
\end{array}\right\} \\
& =\sum_{\{S \subseteq[d], \# S \leq j\}}(-1)^{j-\# S}\left(\begin{array}{l}
d-\# S \\
j-\# S
\end{array}\right) \#\left\{\begin{array}{ll}
\sigma \in S_{d+1}^{l} & \begin{array}{l}
\mathrm{D}^{l}(\sigma) \subseteq S, \\
\sigma(d+1)=d+1-\mu
\end{array}
\end{array}\right\}
\end{aligned}
$$

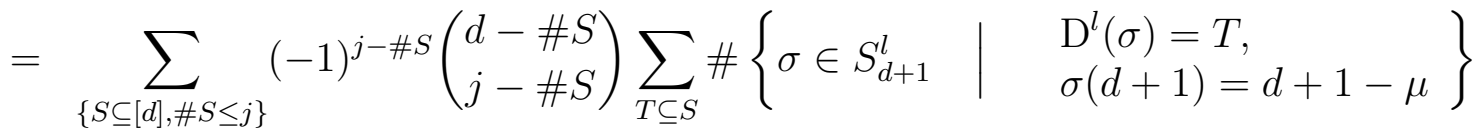

$$
\begin{aligned}
& =\sum_{\{T \subseteq[d], \# T \leq j\}} \#\left\{\begin{array}{l|l}
\sigma \in S_{d+1}^{l} & \begin{array}{l}
\mathrm{D}^{l}(\sigma)=T, \\
\sigma(d+1)=d+1-\mu
\end{array}
\end{array} \sum_{\{S \supseteq T, \# S \leq j\}}(-1)^{j-\# S}\left(\begin{array}{l}
d-\# S \\
j-\# S
\end{array}\right)\right. \\
& =\sum_{\{T \subseteq[d], \# T \leq j\}} \#\left\{\begin{array}{l|l}
\sigma \in S_{d+1}^{l} & \begin{array}{l}
\mathrm{D}^{l}(\sigma)=T, \\
\sigma(d+1)=d+1-\mu
\end{array}
\end{array} \sum_{i=\# T}^{j}(-1)^{j-i}\left(\begin{array}{l}
d-i \\
j-i
\end{array}\right)\left(\begin{array}{l}
d-\# T \\
i-\# T
\end{array}\right) .\right.
\end{aligned}
$$

But

$$
\begin{aligned}
\sum_{i=\# T}^{j}(-1)^{j-i}\left(\begin{array}{l}
d-i \\
j-i
\end{array}\right)\left(\begin{array}{l}
d-\# T \\
i-\# T
\end{array}\right) & =\left(\begin{array}{l}
d-\# T \\
i-\# T
\end{array}\right) \sum_{i=\# T}^{j}(-1)^{j-i}\left(\begin{array}{l}
j-\# T \\
i-\# T
\end{array}\right) \\
& =\delta_{j, \# T} .
\end{aligned}
$$

Hence

$$
\begin{aligned}
& \sum_{k=0}^{d} \sum_{i=0}^{j}(-1)^{j-i}\left(\begin{array}{l}
d-i \\
j-i
\end{array}\right)\left(\begin{array}{l}
d-\mu \\
d-k
\end{array}\right) R(k, i, l) \\
& =\sum_{\{T \subseteq[d], \# T=j\}} \#\left\{\begin{array}{l|l}
\sigma \in S_{d+1}^{l} & \begin{array}{l}
\mathrm{D}^{l}(\sigma)=T, \\
\sigma(d+1)=d+1-\mu
\end{array}
\end{array}\right\} \\
& =\#\left\{\begin{array}{l|l}
\sigma \in S_{d+1}^{l} & \begin{array}{l}
\operatorname{des}^{l}(\sigma)=j, \\
\sigma(d+1)=d+1-\mu
\end{array}
\end{array}\right\} .
\end{aligned}
$$

This completes the proof. 
We note that since for a $(d-1)$-dimensional simplicial complex $\Delta$ and $l \leq d-$ 1 the subdivision operation $\operatorname{sd}^{l}(\bullet)$ is non-trivial in top-dimension it follows from Theorem 5.5. from [4] that iterated application of $\operatorname{sd}^{l}(\bullet)$ will lead to a convergence phenomenon for the $f$-vector. More precisely, for a $(d-1)$-dimensional simplicial complex $\Delta$, set $\Delta^{(n)}:=\underbrace{\operatorname{sd}^{l}\left(\cdots \operatorname{sd}^{l}\right.}_{n}(\Delta)$ and $f^{(n)}(t)=\sum_{i=0}^{d} f_{i-1}^{\Delta^{(n)}} t^{d-i}$ then for $n \rightarrow \infty$ one root of $f^{(n)}(t)$ will go to $-\infty$ and the others converge to complex numbers independent of $\Delta$. This phenomenon was fist observed in [3, Thm. 4.2] for the special case of classical barycentric subdivision $\operatorname{sd}^{1}(\bullet)$. In addition, in [3, Thm. 3.1] it is shown that for $l=1$ the polynomial $f^{(1)}(t)$ has only real roots. Simple examples show that this is not the case for general $l$.

\section{The Transformation Matrices}

For a $(d-1)$-dimensional simplicial complex $\Delta$ we denote by $\mathfrak{H}_{d-1}=\left(h_{i j}^{(d-1)}\right)_{0 \leq i, j \leq d} \in$ $\mathbb{R}^{(d+1) \times(d+1)}$ the matrix of the linear transformation that sends the $h$-vector of $\Delta$ to the $h$-vector of $\operatorname{sd}(\Delta)$ and $\mathfrak{H}_{d-1}^{l}=\left(h_{i j}^{(d-1, l)}\right)_{0 \leq i, j \leq d} \in \mathbb{R}^{(d+1) \times(d+1)}$ the matrix of the transformation of the $h$-vector of $\Delta$ to the $h$-vector of $\operatorname{sd}^{l}(\Delta)$. Thus $\mathfrak{H}_{d-1}^{1}=\mathfrak{H}_{d-1}$. By [3, Thm. 1] we know $h_{i j}^{(d-1)}=A(d+1, i, j+1)$ and more generally by Theorem 3.4 we know $h_{i j}^{(d-1, l)}=A(d+1, i, d+1-j, l)$.

As an illustration we present the matrices $\mathfrak{H}_{d}^{l}$ for $d=4$ and $l=3$ and $l=2$.

$$
\mathfrak{H}_{3}^{3}=\left(\begin{array}{ccccc}
1 & 0 & 0 & 0 & 0 \\
1 & 2 & 1 & 1 & 1 \\
1 & 1 & 2 & 1 & 1 \\
1 & 1 & 1 & 2 & 1 \\
0 & 0 & 0 & 0 & 1
\end{array}\right) \quad \mathfrak{H}_{3}^{2}=\left(\begin{array}{ccccc}
1 & 0 & 0 & 0 & 0 \\
5 & 5 & 5 & 2 & 1 \\
5 & 5 & 6 & 5 & 5 \\
1 & 2 & 3 & 5 & 5 \\
0 & 0 & 0 & 0 & 1
\end{array}\right)
$$

The following lemma follows immediately from the definition of $A(d+1, i, j+1)$.

Lemma 4.1. The sum of all entries of $\mathfrak{H}_{d-1}^{l}$ is given by:

$$
\sum_{0 \leq i, j \leq d} h_{i j}^{(d-1, l)}=\frac{(d+1) !}{l !},
$$

and the sum of all entries of each column is given by:

$$
\sum_{0 \leq j \leq d} h_{i j}^{(d-1, l)}=\frac{d !}{l !}, \quad 0 \leq i \leq d .
$$

The next simple lemma gives an explicit formula for $\mathfrak{H}_{d-1}^{d-1}$ which will serve as the induction base for the proof of monotonocity of the $h$-vector under partial barycentric subdivision in Corollary 4.5 . 
Lemma 4.2. Let $l=d-1$, then the entries of $\mathfrak{H}_{d-1}^{d-1}$ are given by:

$$
h_{i j}^{(d-1, d-1)}=\left\{\begin{array}{l}
0, \quad i=0, j \neq 0 \text { or } i=d, j \neq d \\
2, \quad i=j=1, \ldots, d-1 \\
1, \quad \text { otherwise. }
\end{array}\right.
$$

and hence

$$
\mathfrak{H}_{d-1}^{d-1}=\left(\begin{array}{cccccc}
1 & 0 & 0 & \cdots & 0 & 0 \\
1 & 2 & 1 & \cdots & 1 & 1 \\
1 & 1 & 2 & \cdots & 1 & 1 \\
\vdots & \vdots & \vdots & & \vdots & \vdots \\
1 & 1 & 1 & \cdots & 2 & 1 \\
0 & 0 & 0 & \cdots & 0 & 1
\end{array}\right)
$$

Proof. We will prove it by describing the entries of an arbitrary column. Let $C_{j}=$ $(A(d+1, i, d+1-j, d-1))_{0 \leq i \leq d} \in \mathbb{R}^{(d+1) \times 1}$ be the $j^{\text {th }}$ column of $\mathfrak{H}_{d-1}^{d-1}$. Then by definition the entries of $C_{j}$ count the permutations $\sigma \in S_{d+1}^{d-1}$ such that $\sigma(d+1)=$ $d+1-j$ according to their number of $l$-descents. By fixing the last element, we are left with $d$ permutations $\left\{\sigma_{0}, \sigma_{1}, \ldots, \sigma_{d-1}\right\} \subset S_{d+1}^{d-1}$, and we arrange them in such a way that $\left\{\sigma_{0}(d), \sigma_{1}(d), \ldots, \sigma_{d-1}(d)\right\}$ are in descending order. We count the number of $l$-descents in the following way:

The number of $l$-descents of $\sigma_{i}$ in the first $d-1$ positions is $i$ by the way we have arranged $\sigma_{0}, \ldots, \sigma_{d}$. Moreover since $\sigma_{i}(d+1)=d+1-j$ there is a descent in position $d$ if and only if $i<j$. Therefore, the number of $l$-descents is $i+1$ if $i<j$ and $i$ if $i \geq j$. Now a simple count implies the assertion.

The examples above and the preceding lemma suggest some relations among the entries of $\mathfrak{H}_{d-1}^{l}$ that we verify in the next lemmas.

Lemma 4.3. For $0 \leq i, j \leq d$,

$$
A(d+1, i, d+1-j, l)=A(d+1, d-i, j+1, l) .
$$

Proof. Let us denote by $S_{d+1}^{l}(i, d+1-j)$ the set of permutations $\sigma \in S_{d+1}^{l}$ such that $\operatorname{des}^{l}(\sigma)=i$ and $\sigma(d+1)=d+1-j$. Thus $A(d+1, i, d+1-j, l)=\# S_{d+1}^{l}(i, d+1-j)$. To complete the proof it is enough to provide a bijection between $S_{d+1}^{l}(i, d+1-j)$ and $S_{d+1}^{l}(d-i, j+1)$. Let

$$
\varphi: S_{d+1}^{l}(i, d+1-j) \rightarrow S_{d+1}^{l}(d-i, j+1)
$$

be the map that sends $\sigma=(\sigma(1), \ldots, \sigma(d+1)) \in S_{d+1}^{l}(i, d+1-j)$ to

$$
\varphi(\sigma):=(d+2-\sigma(l), \ldots, d+2-\sigma(1), d+2-\sigma(l+1), \ldots, d+2-\sigma(d+1)) .
$$

Since $\sigma(1), \ldots, \sigma(l)$ are in descending order, we have that $d+2-\sigma(l), \ldots, d+2-$ $\sigma(1)$ are also in descending order and hence $\varphi(\sigma) \in S_{d+1}^{l}$. By definition $\varphi(\sigma)(d+1)=$ $d+1-\sigma(d+1)=d+1-j$. Thus to show that $\varphi(\sigma) \in S_{d+1}^{l}(d-i, j+1)$ it remains to verify that the number of $l$-descents of $\varphi(\sigma)$ is $d-i$. 
We show that $m \in[d]$ is an $l$-descent of $\sigma$ if and only if $m$ is not an $l$-descent of $\varphi(\sigma)$. If $m \in[l]$ then $\sigma(j)>\sigma(l+1)$ implies $d+2-\sigma(j)<d+2-\sigma(l+1)$ and $\sigma(j)<\sigma(l+1)$ implies $d+2-\sigma(j)>d+2-\sigma(l+1)$. Analogously, if $m \in[d] \backslash[l]$ then $\sigma(m)>\sigma(m+1)$ implies $d+2-\sigma(m)<d+2-\sigma(m+1)$ and $\sigma(m)<\sigma(m+1)$ implies $d+2-\sigma(m)>d+2-\sigma(m+1)$.

Therefore, the number of $l$-descents of $\varphi(\sigma)$ is $d-i$. This completes the proof since $\varphi$ is clearly a bijection.

Proposition 4.4. For $0 \leq i, j \leq d$,

$$
A(d+1, i, d+1-j, l+1) \leq A(d+1, i, d+1-j, l) .
$$

In addition, for $d \geq 4,0 \leq j \leq d$ and $2 \leq i \leq d-2$, inequality (2) is strict.

Proof. For the sake of short notation, within the proof we say descent for corresponding $l$-descent and "^" means that the entry is missing in the permutation. We define a map

$$
\psi: S_{d+1}^{l+1}(i, d+1-j) \rightarrow S_{d+1}^{l}(i, d+1-j)
$$

as follows:

Let $\sigma \in S_{d+1}^{l+1}(i, d+1-j)$ be a permutation for which $p$ is the number of descents in the first $l+1$ positions and $i-p$ descents in the remaining positions for some $0 \leq p \leq l$. Thus we can write $\sigma=(\sigma(1), \ldots, \sigma(p), \ldots, \sigma(l+1), \sigma(l+2), \ldots, \sigma(d+1))$ such that $\sigma(p)>\sigma(l+2)$ and $\sigma(p+1)<\sigma(l+2)$, where $\sigma(1), \ldots, \sigma(l+1)$ are in descending order and $\sigma(d+1)=d+1-j$.

We define

$$
\psi(\sigma)=(\sigma(1), \ldots, \hat{\sigma}(p+1), \ldots, \sigma(l+1), \sigma(p+1), \ldots, \sigma(d)) ;
$$

i.e we change the position of $\sigma(p+1)$ from $p+1$ to $l+1$. It is easy to see that $\psi(\sigma)$ is a permutation for which $p$ is the number of descents in the first $l$ position and $i-p$ descents in the remaining position with $\psi(\sigma)(d)=d+1-j$. Therefore $\psi(\sigma) \in S_{d+1}^{l}(i, d+1-j)$. Clearly, $\psi$ is injective and hence we have $S_{d+1}^{l+1}(i, d+1-j) \subseteq$ $S_{d+1}^{l}(i, d+1-j)$ which implies $A(d+1, i, d+1-j, l+1) \leq A(d+1, i, d+1-j, l)$.

Now assume $d \geq 4,0 \leq j \leq d$ and $2 \leq i \leq d-2$. For the proof of the strict inequality in (2) it suffices to find at least one element $\sigma \in S_{d+1}^{l}(i, d+1-j)$ that does not have a preimage under $\psi$ in $S_{d+1}^{l+1}(i, d+1-j)$. We consider two cases:

Case $1(j \neq 0)$ : We set $\sigma(d+1)=d+1-j$ and $\sigma(d)=d+1$. Now we are left with $d-1$ elements to be arranged with $i-1$ descents. Let $\rho_{1}, \ldots, \rho_{d-1}$ be the remaining elements of $[d+1]$ arranged in ascending order; i.e. $\rho_{s}<\rho_{t}$ for $s<t$. We have further two possibilities:

(a) $i-1 \leq l$. Then reordering the elements as

$$
\rho_{l+1}, \ldots, \hat{\rho}_{l+2-i}, \ldots, \rho_{1}, \rho_{l+2-i}, \rho_{l+2}, \ldots, \rho_{d-1}
$$

yields the required number of $l$-descents. Clearly, the formal preimage of $\sigma$ under $\psi$ has not belong to $S_{d+1}^{l+1}(i, d+1-j)$ since it has only one descent but $i \geq 2$. 
(b) $i-1>l$. Then we reorder the elements in the first $l+1$ positions as

$$
\rho_{l+1}, \ldots, \rho_{2}, \rho_{1},
$$

which contributes $l$ to the number of descents. The remaining $d-1-(l+1)$ elements can be arranged in such a way that they contribute $i-1-l$ to the number of descents. By this setting we cannot have a descent at $(l+1)^{\text {th }}$ and $(d-1)^{\text {st }}$ position, so at most we can have $d-2$ descents which coincides with the upper bound for $i$. Again, the formal preimage of $\sigma$ under $\psi$ does not belong to $S_{d+1}^{l+1}(i, d+1-j)$ since its number of descents are $i-l$.

Case $2(j=0)$ : We set $\sigma(d+1):=d+1$. Thus we are left with the $d$ elements $[d]$ to be arranged yielding a permutation in $S_{d+1}^{l}(i, d+1-l)$. The same arrangement as in Case 1 with $d$ elements and $i$ descents will give us the required element $\sigma$.

As a consequence of Theorem 3.4 and Proposition 4.4 we can deduce a result on the growth of the $h$-vector under $l^{\text {th }}$ partial barycentric subdivision.

Corollary 4.5. Let $\Delta$ be a d-dimensional simplicial complex such that $h_{i}^{\Delta} \geq 0$ for all $0 \leq i \leq d$. Then $h_{i}^{\Delta} \leq h_{i}^{\text {sd }^{l}(\Delta)}$ for $0 \leq i \leq d$.

Proof. It is easy to see that $h_{0}^{\mathrm{sd}^{l}(\Delta)}=h_{0}^{\Delta}$ and $h_{d}^{\mathrm{sd}^{l}(\Delta)}=h_{d}^{\Delta}$, thus we are left with the case $1 \leq i \leq d-1$. Since by Theorem $3.4 h_{i}^{\mathrm{sd}^{l}(\Delta)}$ is a non-negative linear combination of the $h_{j}^{\Delta}$ it suffices to show that the entries of the submatrix $\left(h_{i j}^{(d-1, l)}\right)_{1 \leq i \leq d-1,0 \leq j \leq d}$ are non-zero. Again, by equation 2 it is enough to consider the case $l \stackrel{l \leq l \leq d}{=}-1$. By Lemma 4.2 we complete the proof.

The consequence of the preceding corollary for the smaller class of Cohen-Macaulay simplicial complexes also follows from a very general result by Stanley [8, Theorem 4.10] using the fact that $l^{\text {th }}$ partial barycentric subdivision is a quasi-geometric subdivision. Note that $h_{i}^{\Delta} \geq 0$ for Cohen-Macaulay simplicial complexes.

Let $\mathfrak{F}_{d-1}$ be the matrix of the transformation that sends $f$-vector of $\Delta$ to $f$-vector of $\operatorname{sd}(\Delta)$. We denote by $\mathfrak{F}_{d-1}^{l}$ the matrix of the transformation from the $f$-vector of $\Delta$ to the $f$-vector of $\operatorname{sd}^{l}(\Delta)$. Both matrices $\mathfrak{F}_{d-1}$ and $\mathfrak{F}_{d-1}^{l}$ are square matrices of order $d+1$, with $\mathfrak{F}_{d-1}^{1}=\mathfrak{F}_{d-1}$. By Theorem 3.1] the entries of $\mathfrak{F}_{d-1}^{l}=\left(f_{i j}^{(d-1, l)}\right)_{0 \leq i, j \leq d}$ are given by $f_{i j}^{(d-1, l)}=R(j, i, l)$.

The following lemma shows that the matrices $\mathfrak{F}_{d-1}^{l}$ and $\mathfrak{H}_{d-1}^{l}$ are diagonalizable.

Proposition 4.6. For $1 \leq l \leq d-1$ :

(1) The matrices $\mathfrak{F}_{d-1}^{l}$ and $\mathfrak{H}_{d-1}^{l}$ are similar.

(2) The matrices $\mathfrak{F}_{d-1}^{l}$ and $\mathfrak{H}_{d-1}^{l}$ are diagonalizable with eigenvalue 1 of multiplicity $l+1$ and eigenvalues $\frac{(l+1) !}{l !}, \ldots, \frac{d !}{l !}$ of multiplicity 1 .

Proof. - Since the transformation sending the $f$-vector of a simplicial complex to the $h$-vector of a simplicial complex is an invertible linear transformation, the first assertion follows. 
- Clearly, $\mathfrak{F}_{d-1}^{l}$ is an upper triangular matrix with diagonal entries

$$
\underbrace{1, \ldots, 1}_{(l+1) \text {-times }}, \frac{(l+1) !}{l !}, \ldots, \frac{d !}{l !} .
$$

Let $\left(\mathfrak{F}_{d-1}^{l}\right)^{\perp}$ be the transpose of $\mathfrak{F}_{d-1}^{l}$. Then for $\left(\mathfrak{F}_{d-1}^{l}\right)^{\perp}$, the first $(l+1)$ unit vectors are eigenvectors for the eigenvalue 1 . Also, The eigenvalues $\frac{(l+1) !}{l !}, \ldots, \frac{d !}{l !}$ are pairwise different. This implies that $\left(\mathfrak{F}_{d-1}^{l}\right)^{\perp}$ is diagonalizable. But then $\mathfrak{F}_{d-1}^{l}$ is diagonalizable.

Corollary 4.7. Let $\nu=\left(\nu_{0}, \ldots, \nu_{d}\right)$ be an eigenvector of the matrix $\mathfrak{H}_{d-1}^{l}$ for the eigenvalue $\lambda$ such that $\lambda \neq \frac{d !}{l !}$. Then $\sum_{i=0}^{d} \nu_{i}=0$.

Proof. Since

$$
\begin{aligned}
\mathfrak{H}_{d-1}^{l} \nu & =\lambda \nu \\
\Rightarrow(1, \ldots, 1) \mathfrak{H}_{d-1}^{l} \nu & =(1, \ldots, 1) \lambda \nu
\end{aligned}
$$

But by Lemma 4.1, $(1, \ldots, 1) \mathfrak{H}_{d-1}^{l}=\frac{d !}{l !}(1, \ldots, 1)$. Therefore, either $\lambda=\frac{d !}{l !}$ or $\sum_{i=0}^{d} \nu_{i}=0$. Since $\lambda \neq \frac{d !}{l !}$ we are done.

Next we try to gain a better understanding of the eigenvectors of $\mathfrak{H}_{d-1}^{l}$.

Lemma 4.8. Let $d \geq 2$ and $\nu_{1}^{(1)}, \ldots, \nu_{1}^{(l+1)}, \nu_{l+1}, \ldots, \nu_{d}$ be a basis of eigenvectors of the matrix $\mathfrak{F}_{d-1}^{l}$, where $\nu_{1}^{(1)}, \ldots, \nu_{1}^{(l+1)}$ are eigenvectors for the eigenvalue 1 and $\nu_{l+1}, \ldots, \nu_{d}$ are eigenvectors for the eigenvalues $\{l+1, \ldots, d\}$, respectively. Then $\left(\nu_{1}^{(1)}, 0\right), \ldots,\left(\nu_{1}^{(l+1)}, 0\right),\left(\nu_{l+1}, 0\right), \ldots,\left(\nu_{d}, 0\right)$ are eigenvectors of the matrix $\mathfrak{F}_{d}^{l}$ for the eigenvalues $\{\underbrace{1, \ldots, 1}_{(l+1) \text { times }}, l+1, \ldots, d\}$.

Proof. Since both $\mathfrak{F}_{d-1}^{l}$ and $\mathfrak{F}_{d}^{l}$ are upper triangular and $\mathfrak{F}_{d-1}^{l}$ is obtained by deleting $(d+2)^{\text {nd }}$ column and row from $\mathfrak{F}_{d}^{l}$ the assertion follows.

Let $\hat{\mathfrak{H}}_{d-1}^{l}$ be a matrix obtained be deleting the first and last rows and columns of $\mathfrak{H}_{d-1}^{l}$. Thus $\hat{\mathfrak{H}}_{d-1}^{l}$ is a $d$ by $d$ square matrix.

Lemma 4.9. The matrix $\hat{\mathfrak{H}}_{d-1}^{l}$ is diagonalizable.

Proof. By definition and 3.4 the first row of $\mathfrak{H}_{d-1}^{l}$ is the first unit vector and the last row of $\mathfrak{H}_{d-1}^{l}$ is the $(d+1)^{\text {st }}$ unit vector. Thus the characteristic polynomial of $\mathfrak{H}_{d-1}^{l}$ splits into $(1-t)^{2}$ times the characteristic polynomial of $\hat{\mathfrak{H}}_{d-1}^{l}$. Therefore, $\hat{\mathfrak{H}}_{d-1}^{l}$ has eigenvalues

$$
\underbrace{1, \ldots, 1}_{(l-1)-\text { times }}, \frac{(l+1) !}{l !}, \ldots, \frac{d !}{l !} .
$$

To show the matrix $\hat{\mathfrak{H}}_{d-1}^{l}$ is diagonalizable, it is enough to show that the eigenspace for the eigenvalue 1 is of dimension $l-1$. 
For this we again consider the full matrix $\mathfrak{H}_{d-1}^{l}$. Since $\mathfrak{H}_{d-1}^{l}$ is diagonalizable there is a basis $\omega_{1}^{(1)}, \ldots, \omega_{1}^{(l+1)}, \omega_{l+1}, \ldots, \omega_{d}$ of $\mathbb{R}^{d+1}$ consisting of eigenvectors of $\mathfrak{H}_{d-1}^{l}$. We can choose the numbering such that $\omega_{1}^{(i)}, 1 \leq i \leq l+1$ are eigenvectors for the eigenvalue 1 and $\omega_{j}$ is an eigenvector for the eigenvalues $\frac{j !}{l !}, l+1 \leq j \leq d$, respectively.

Again, since the first and last row of $\mathfrak{H}_{d-1}^{l}$ are the first and $(d+1)^{\text {st }}$ unit vector we can choose the eigenvectors of $\mathfrak{H}_{d-1}^{l}$ for the eigenvalue $\lambda=1$ as follows: $\omega_{1}^{(1)}$ and $\omega_{1}^{(2)}$ can be chosen such that

$$
\omega_{1}^{(1)}=\left(1, k_{11}, \ldots, k_{1(d-1)}, 0\right) \text { and } \omega_{1}^{(2)}=\left(0, k_{21}, \ldots, k_{2(d-1)}, 1\right),
$$

and $\omega_{1}^{(i)}$ can be chosen such that $\omega_{1}^{(i)}=\left(0, k_{i 1}, \ldots, k_{i(d-1)}, 0\right)$ for $3 \leq i \leq l+1$. Clearly, this implies that deleting the leading and trailing 0 form the $\omega_{1}^{(i)}$ for $3 \leq$ $i \leq l+1$ yields eigenvectors $\hat{\omega}_{1}{ }^{(i)}=\left(k_{i 1}, \ldots, k_{i(d-1)}\right)$ of $\hat{\mathfrak{H}}_{d-1}^{l}$ for the eigenvalue $\lambda=1$. Obviously, the set of vectors $\left\{\hat{\omega}_{1}{ }^{(3)}, \ldots, \hat{\omega}_{1}{ }^{(l+1)}\right\}$ is linearly independent. Hence we have shown that the dimension of the eigenspace for the eigenvalue 1 of $\hat{\mathfrak{H}}_{d-1}^{l} l-1$.

The above lemma is a key ingredient in proving the following theorem.

Theorem 4.10. Let $d \geq 2$ and let $\omega_{1}^{(1)}, \ldots, \omega_{1}^{(l+1)}, \omega_{l+1}, \ldots, \omega_{d}$ be a basis of eigenvectors of the matrix $\mathfrak{H}_{d-1}^{l}$, where $\omega_{1}^{(i)}, 1 \leq i \leq l+1$ are eigenvectors for the eigenvalue 1 and $\omega_{j}$ is an eigenvector for the eigenvalue $\frac{j !}{l !}, l+1 \leq j \leq d$.

(1) Let $\Delta$ be a $(d-1)$-dimensional simplicial complex. If we expand h-vector of $\Delta$ in terms of eigenvectors of the matrix $\mathfrak{H}_{d-1}^{l}$, the coefficient of the eigenvector for the eigenvalue $\frac{d !}{l !}$ is non-zero.

(2) The first and the last coordinate entry in $\omega_{1}^{(3)}, \ldots, \omega_{1}^{(l+1)}, \omega_{l+1}, \ldots, \omega_{d}$ is zero.

(3) The vectors $\omega_{1}^{(1)}$ and $\omega_{1}^{(2)}$ can be chosen such that

$$
\omega_{1}^{(1)}=\left(1, i_{1}, \ldots, i_{d-1}, 0\right) \text { and } \omega_{1}^{(2)}=\left(0, j_{1}, \ldots, j_{d-1}, 1\right) .
$$

(4) The vector $\omega_{d}$ can be chosen such that $\omega_{d}=\left(0, b_{1}, \ldots, b_{d-1}, 0\right)$ for strictly positive rational numbers $b_{i}, 1 \leq i \leq d-1$.

Proof. Let us expand the $f$-vector of $\Delta$ in terms of a basis of eigenvectors of the matrix $\mathfrak{F}_{d-1}^{l}$. Since $f_{d-1}^{\Delta} \neq 0$ from Lemma 4.8 we deduce that the coefficient of the eigenvector for the highest eigenvalue is non-zero. Since $\mathfrak{F}_{d-1}^{l}$ and $\mathfrak{H}_{d-1}^{l}$ are similar so (1) follows.

Assertions (2) and (3) immediately follow from the proof of Lemma 4.9.

For (4) consider the matrix $\hat{\mathfrak{H}}_{d-1}^{l}$ as defined above. It is easily seen that the entries of $\hat{\mathfrak{H}}_{d-1}^{l}$ are strictly positive numbers. Therefore, by the Perron-Frobenius Theorem [6] it follows that there is an eigenvector $\hat{\omega}_{d}^{l}$ for the eigenvalue $\frac{d !}{l !}$ with strictly positive entries. Hence $\left(0, \hat{\omega}_{d}^{l}, 0\right)$ is the required eigenvector. 


\section{Open Problems}

In this section we discuss some of the open problems related to the above work. Corollary 4.7 describes properties of the eigenvectors of the matrix $\mathfrak{H}_{d-1}^{l}$ for the eigenvalue $\lambda$ such that $\lambda \neq \frac{d !}{l !}$. For the eigenvalue $\lambda=\frac{d !}{l !}$ we were able to deduce its non-negativity in Theorem 4.10 (4) but were not able to give more structural results or even provide an explicit description. By [4] when applying $l^{\text {th }}$ partial barycentric subdivision iteratively the limiting behavior of the $h$-vector is determined by this eigenvector, in a sense specified in [4]. Hence some information can be read off from [4] nevertheless complete information about that eigenvector would be desirable.

For example, for $d=4$ we have following eigenvectors, corresponding to the eigenvalues $\frac{4 !}{3 !}, \frac{4 !}{2 !}, \frac{4 !}{1 !}$, respectively.

$$
\left(\begin{array}{l}
0 \\
1 \\
1 \\
1 \\
0
\end{array}\right),\left(\begin{array}{l}
0 \\
1 \\
\frac{5}{3} \\
1 \\
0
\end{array}\right),\left(\begin{array}{l}
0 \\
1 \\
\frac{7}{2} \\
1 \\
0
\end{array}\right)
$$

For $d=5$ we have the following eigenvectors, corresponding to the eigenvalues $\frac{5 !}{4 !}, \frac{5 !}{3 !}, \frac{5 !}{2 !}, \frac{5 !}{1 !}$, respectively.

$$
\left(\begin{array}{l}
0 \\
1 \\
1 \\
1 \\
1 \\
0
\end{array}\right),\left(\begin{array}{c}
0 \\
1 \\
\frac{12}{7} \\
\frac{12}{7} \\
1 \\
0
\end{array}\right),\left(\begin{array}{c}
0 \\
1 \\
\frac{46}{11} \\
\frac{46}{11} \\
1 \\
0
\end{array}\right),\left(\begin{array}{c}
0 \\
1 \\
\frac{17}{2} \\
\frac{17}{2} \\
1 \\
0
\end{array}\right) .
$$

Similarly, for $d=6$ we have following eigenvectors, corresponding to the eigenvalues $\frac{6 !}{5 !}, \frac{6 !}{4 !}, \frac{6 !}{3 !}, \frac{6 !}{2 !}, \frac{6 !}{1 !}$, respectively.

$$
\left(\begin{array}{l}
0 \\
1 \\
1 \\
1 \\
1 \\
1 \\
0
\end{array}\right),\left(\begin{array}{l}
0 \\
1 \\
\frac{7}{4} \\
\frac{7}{4} \\
\frac{7}{4} \\
1 \\
0
\end{array}\right),\left(\begin{array}{c}
0 \\
1 \\
\frac{1941}{437} \\
\frac{2146}{437} \\
\frac{1941}{437} \\
1 \\
0
\end{array}\right),\left(\begin{array}{c}
0 \\
1 \\
\frac{5431}{527} \\
\frac{8906}{527} \\
\frac{5431}{527} \\
1 \\
0
\end{array}\right),\left(\begin{array}{c}
0 \\
1 \\
\frac{586}{33} \\
\frac{5459}{132} \\
\frac{586}{33} \\
1 \\
0
\end{array}\right) .
$$

Thus the following problem appears to be interesting.

Problem 5.1. Give a description of eigenvectors of the matrices $\mathfrak{F}_{d-1}^{l}$ and $\mathfrak{H}_{d-1}^{l}$ for the eigenvalue $\frac{d !}{l !}$.

Let $V$ be a vertex set such that $\# V=d$ and let $2^{V}$ denote the simplex with vertex set $V$. Let $\Gamma$ be the first barycentric subdivision of $2^{V}$. The $h$-polynomial 
$h(\Gamma, x)=\sum_{i=0}^{d} h_{i} x^{d-i}$ of $\Gamma$ has the following combinatorial interpretation.

$$
h(\Gamma, x)=\sum_{\sigma \in S_{d}} x^{\operatorname{des}(\sigma)}=\sum_{\sigma \in S_{d}} x^{\operatorname{ex}(\sigma)},
$$

where $\operatorname{ex}(\sigma)$ denotes the number of excedances of $\sigma$, defined by

$$
\operatorname{ex}(\sigma)=\#\{i \mid \sigma(i)>i\}
$$

The first equality follows from [9, Theorem 3.13.1] (it is also a consequence of [3, Thm 1] and Theorem 3.4), and the second is a consequence of [9, Proposition 1.4.3]. In [8], the local $h$-polynomial $\ell_{V}(\Gamma, x)$ of $\Gamma$ has been defined and given in a similar passion of equation (3) as follows:

$$
\ell_{V}(\Gamma, x)=\sum_{\sigma \in D_{d}} x^{\operatorname{ex}(\sigma)}
$$

where $D_{d}$ denotes the set of all derangements in $S_{d}$. We suggest the followings:

Problem 5.2. Give an interpretation of local h-polynomial for the $l^{\text {th }}$ partial barycentric subdivision similar to (41) in terms of a suitably defined l-excedance statistic on a newly defined set of l-derangements satisfying an analog of (3)

Already the question of finding a statistic on $S_{d}^{l}$ fulfilling a statement analogous to (3) seems to be hard and challenging. We note that in [1] a theory of local $\gamma$-vectors of subdivisions was initiated.

Problem 5.3. Define an l-excedance statistic on $S_{d}^{l}$ such that the l-excedance and l-descent statistic on $S_{d}^{l}$ are equally distributed; i.e. satisfy an analog of (4).

For Problem 5.3 we tried, different approaches. Despite not yielding a solution to the problem the following idea resulted in some interesting data. We define an injective map say $\chi: S_{d}^{l} \rightarrow S_{d}$ in the following way. Let $\sigma \in S_{d}^{l}$ such that $\sigma=(\sigma(1), \sigma(2), \ldots, \sigma(d))$, with first $l$ elements are in descending order, then:

$$
\chi(\sigma)= \begin{cases}(\sigma(l), \ldots, \sigma(1), \sigma(l+1), \ldots, \sigma(d)), & \text { if } \sigma(l+1)>\sigma(1) \\ \left(\sigma\left(l_{1}\right), \ldots, \sigma(1), \sigma(l), \ldots, \sigma\left(l_{1}+1\right),\right. & \text { or } \sigma(l)>\sigma(l+1) ; \\ \sigma(l+1), \ldots, \sigma(d)), & \text { if } \sigma\left(l_{1}\right)>\sigma(l+1) \\ & \text { and } \sigma\left(l_{1}+1\right)<\sigma(l+1) .\end{cases}
$$

Now define the number of $l$-excedances $\operatorname{ex}^{l}(\sigma)$ of $\sigma \in S_{d}^{l}$ to be number of usual excedances of $\chi(\sigma)$, i.e.

$$
\operatorname{ex}^{l}(\sigma):=\#\{i \mid \chi(\omega)(i)>i\} \text {. }
$$

We apply this definition for different values of $d$ and $l$. For a fixed $d$, the $l$-descent and $l$-excedance statistic are equally distributed on $S_{d}^{l}$ for $l=d-1$ and $l=d-2$. But for other values of $l$ the two statistics appear to be different. Nevertheless, the obtained data has some surprising and unexplained symmetry. For example, for $S_{5}^{l}$ we have following tables for the number of $l$-descents, 


\begin{tabular}{|l|c|c|c|c|}
\cline { 3 - 4 } \multicolumn{1}{c|}{} & \multicolumn{4}{|c|}{$l=$} \\
\# of l-descents $=$ & & 4 & 3 & 2 \\
\cline { 3 - 5 } & 1 & 1 & 1 \\
\cline { 3 - 5 } & 1 & 6 & 16 \\
\cline { 3 - 5 } & 2 & 1 & 6 & 26 \\
\cline { 3 - 5 } & 3 & 1 & 6 & 16 \\
\cline { 3 - 5 } & 1 & 1 & 1 \\
\hline
\end{tabular}

and the following table for the number of $l$-excedances.

\begin{tabular}{|c|c|c|c|c|}
\hline & & \multicolumn{3}{|c|}{$l=$} \\
\hline & & 4 & 3 & 2 \\
\hline \multirow{5}{*}{$\#$ of $l$-excedances $=$} & 0 & 1 & 1 & 1 \\
\hline & 1 & 1 & 6 & 14 \\
\hline & 2 & 1 & 6 & 30 \\
\hline & 3 & 1 & 6 & 14 \\
\hline & 4 & 1 & 1 & 1 \\
\hline
\end{tabular}

Similarly, for $S_{6}^{l}$ the number of $l$-descents are shown in the following table,

\begin{tabular}{|c|c|c|c|c|c|}
\hline & \multicolumn{4}{|c|}{$l=$} \\
\hline & & 5 & 4 & 3 & 2 \\
\hline \multirow{6}{*}{$\#$ of $l$-descents $=$} & 0 & 1 & 1 & 1 & 1 \\
\hline & 1 & 1 & 7 & 22 & 42 \\
\hline & 2 & 1 & 7 & 37 & 137 \\
\hline & 3 & 1 & 7 & 37 & 137 \\
\hline & 4 & 1 & 7 & 22 & 42 \\
\hline & 5 & 1 & 1 & 1 & 1 \\
\hline
\end{tabular}

and the number of $l$-excedances are shown in the following table.

\begin{tabular}{|c|c|c|c|c|c|}
\hline & & \multicolumn{4}{|c|}{$l=$} \\
\hline & & 5 & 4 & 3 & 2 \\
\hline \multirow{6}{*}{$\#$ of $l$-excedances $=$} & 0 & 1 & 1 & 1 & 1 \\
\hline & 1 & 1 & 7 & 17 & 33 \\
\hline & 2 & 1 & 7 & 42 & 146 \\
\hline & 3 & 1 & 7 & 42 & 146 \\
\hline & 4 & 1 & 7 & 17 & 33 \\
\hline & 5 & 1 & 1 & 1 & 1 \\
\hline
\end{tabular}

We close by briefly mentioning an interesting problem relating to the $\gamma$-vector introduced by Gal [5]. The behavior of the $\gamma$-vector under barycentric subdivision was studied in [7]. In [1] a theory of local $\gamma$-vectors are started and in [2, Theorem 1.5] a nice interpretation of the local $\gamma$-vector was given for the classical barycentric subdivision. Again one can ask the same questions for the $\gamma$ and local $\gamma$-vector of the $l^{\text {th }}$ partial barycentric subdivision.

\section{REFERENCES}

[1] C.A. Athenasiadis, Flag subdivisions and $\gamma$-vectors, http://arxiv.org/abs/1106.4520.

[2] C.A. Athanasiadis, C. Savvidou, The local h-vector of the cluster subdivision of a simplex, Seminaire Lotharingien de Combinatoire 66 (2012), Article B66c, 21pp. 
[3] F. Brenti, V. Welker, f-vectors of barycentric subdivisions, Math. Z. 259 (2008) 849-865.

[4] E. Delucchi, L. Sabalka, A. Pixton, f-polynomials of subdivisions of complexes Discrete Math. to appear, http://arxiv.org/abs/1002.3201.

[5] S.R. Gal, Real root conjecture fails for five- and higher-dimensional spheres, Discrete Comput. Geom. 34 (2005), 269-284.

[6] R. A. Horn, C. R. Johnson, Matrix Analysis, Cambridge University Press, Cambridge (1985).

[7] E. Nevo, T.K. Petersen, B. Tenner, The $\gamma$-vector of a barycentric subdivision, J. Combin. Theory Ser. A 118 (2011), 1364-1380.

[8] R. P. Stanley, Subdivision and local h-vectors, J. Amer. Math. Soc. 5 (1992) 805-851.

[9] R. P. Stanley, Enumerative Combinatorics, Vol. 1, Second edition, Cambridge University Press, 2012.

COMSATS Institute of Information Technology, Lahore, Pakistan

E-mail address: sarfrazahmad@ciitlahore.edu.pk

Fachbereich Mathematik und Information, Philipps-Universität Marburg, 35032 Marburg, Germany

E-mail address: welker@mathematik.uni-marburg.de 